\title{
Residual Effects of Sleep Medications Are Commonly Reported and Associated with Impaired Patient-Reported Outcomes among Insomnia Patients in the United States
}

\author{
Timothy Fitzgerald ${ }^{1}$ and Jeffrey Vietri $^{2}$ \\ ${ }^{1}$ Merck \& Co., Whitehouse Station, NJ 08889, USA \\ ${ }^{2}$ Kantar Health, 20121 Milan, Italy \\ Correspondence should be addressed to Jeffrey Vietri; jeffrey.vietri@kantarhealth.com
}

Received 12 August 2015; Accepted 15 November 2015

Academic Editor: Giora Pillar

Copyright (C) 2015 T. Fitzgerald and J. Vietri. This is an open access article distributed under the Creative Commons Attribution License, which permits unrestricted use, distribution, and reproduction in any medium, provided the original work is properly cited.

\begin{abstract}
Study Objective. To measure the association of symptoms attributed to residual effects of sleep medication (e.g., drowsiness, difficulty concentrating, and impaired memory) on self-reported functioning and satisfaction with these medications. Methods. Individuals using prescription medications for insomnia were invited to complete an Internet-based survey. Respondents were compared according to the presence of self-reported residual effects; relationships between severity of these effects and outcomes were modeled using regression. Measures included the Brief Insomnia Questionnaire, Work Productivity and Activity Impairment Questionnaire, and SATMED-Q. Subgroup analyses were conducted with patients aged $\geq 65$ years. Approximately $80 \%$ reported experiencing $\geq 1$ residual effect. The severity of residual effects was associated with increased residual effect-related work impairment, including absenteeism $(\mathrm{RR}=1.46, p<0.001)$, presenteeism $(\mathrm{RR}=1.12, p<0.001)$, overall work impairment $(\mathrm{RR}=1.13, p<0.001)$, and nonwork activity impairment $(\mathrm{RR}=1.11, p<0.001)$. More severe residual symptoms were also associated with increased difficulty in home management $($ Beta $=.31, p<0.001)$, ability to work (Beta $=.31, p<0.001)$, social relationships, $($ Beta $=.32, p<0.001)$, close personal relationships (Beta $=.30, p<0.001$ ), and lower medication satisfaction (Beta $=-.37, p<0.001)$. Conclusions. Individuals using medications for insomnia commonly experience symptoms considered as residual effects, and these symptoms are associated with greater interference of sleep-related problems at work, at home, and with social relationships.
\end{abstract}

\section{Introduction}

Insomnia is a debilitating condition that accompanies several sleep, medical, and psychiatric disorders. It is diagnosed via subjective reports of persistent difficulty falling asleep, staying asleep, and/or experiencing sleep of poor quality. Insomnia confers significant daytime symptoms such as fatigue, low energy, impaired cognitive functioning, mood disturbance, and perceptions of decreased global functioning. In fact, daytime symptoms are those which most frequently lead patients to seek treatment [1]. Acute insomnia regularly occurs with life events or sleep schedule changes. For some, however, insomnia becomes unrelenting and chronic. Overall, insomnia has become a prevalent and costly public health concern, associated with long-term effects on functioning and quality of life.

Approximately $25 \%$ of U.S. adults report dissatisfaction with their sleep, $10-15 \%$ report insomnia symptoms, and 6$10 \%$ have an insomnia disorder [2]. Population-based longitudinal data show nearly $70 \%$ of patients with baseline insomnia also report insomnia one year later, and $50 \%$ of those with baseline insomnia continue to report insomnia up to three years later [3, 4]. In fact, insomnia has become one of the most prevalent complaints in the primary care setting [5]. Moreover, high rates of comorbidity between insomnia and medical/psychiatric disorders have been described. Odds ratios reported by the 2002 U.S. National Health Interview Survey and the U.S. National Comorbidity Survey showed those with 
insomnia to be twice as likely to present with congestive heart failure and up to five times more likely to present with a psychiatric disorder [6,7]. Further, insomnia is strongly associated with hypertension and pain conditions, as well as greater risk of mortality, specifically in older adults [8-10].

Treatment can consist of behavioral/psychological interventions and/or pharmacotherapy. In general, it is considered that primary insomnia and secondary insomnia respond to both pharmacotherapy and behavioral/psychological intervention $[11,12]$. Common agents to treat insomnia include over-the-counter agents (OTCs, antihistamines, melatonin, and herbal preparations), prescription hypnotic drugs (benzodiazepines, BzRAs, chronobiotic agents, and low-dose doxepin hydrochloride), and other prescription agents not specifically indicated for insomnia (antidepressants, antipsychotics, and anticonvulsants) [13]. However, sleep-promoting agents can produce adverse effects, particularly in the elderly [13]. Residual sleep medication effects have the potential to interfere with quality of life and include drowsiness, difficulty concentrating, headaches, nausea, dry mouth, oversleeping, and nightmares. Memory can also be affected, including impaired short-term memory and periods of amnesia reported in the literature [14-16].

Of the hypnotics, benzodiazepines and nonbenzodiazepine hypnotics with longer half-lives tend to produce residual impairment or "hang over," particularly with middle-of-the-night dosing and regular use $[17,18]$. Residual effects of hypnotics include sedation, cognitive impairment, motor incoordination, ataxia, dizziness, and gastrointestinal upset. In the elderly, the use of sedating drugs is dangerously associated with increased fall risk [19]. Meanwhile, the use of some antidepressants to treat insomnia has been associated with increased suicidal ideation, increased mania/hypomania in patients with bipolar disorder, and exacerbation of restless legs syndrome [20]. Further, the use of anticonvulsants (e.g., pregabalin) can produce daytime sedation, dizziness, and cognitive impairment [13]. Finally, the use of antipsychotics has been correlated with exacerbation of restless legs syndrome and increased mortality, particularly in elderly individuals [11]. From the standpoint of concern for public safety, insomnia treatments have been shown to impair nextday driving and increase the risk of motor vehicle accidents, particularly in women [21].

As above, older patients are particularly vulnerable to residual sleep medication effects. Meta-analysis of patients aged 60 years and older, who were free of other psychiatric disorders $(N=2,417)$, showed significantly higher odds of adverse cognitive events, adverse psychomotor effects, and daytime fatigue when patients used any hypnotic sedative, compared with placebo [22]. A large $(N=15,528)$ study of nursing home patients (mean age $=81$ ), with hip fracture documented in Medicare Part A and Part D fee-for-service claims, showed elevated risk of hip fracture among users of a nonbenzodiazepine hypnotic sleep drug. Effects were particularly strong in new users [23].

Ultimately, residual sleep medication effects are associated with impaired functioning and lowered quality of life in insomnia patients and confer safety risks for both the patients and the public. Moreover, the literature reports older patients to be at heightened risk for adverse side effects [13]. Little research, however, has characterized the impact of adverse side effects across multiple domains of functioning in the same group of patients-particularly in the elderly. The current study was conducted to collect information on the relationship between self-reported residual effects of prescription sleep medications and patient-reported outcomes. A secondary objective of the study was to describe the relationship between these symptoms and outcomes in users of these medications aged 65 years and older, since, as described above, these patients may be particularly vulnerable to residual effects [13].

\section{Materials and Methods}

The present study was a cross-sectional survey of current and former users of prescription medications for sleep reporting a diagnosis of insomnia $(N=2,250)$. For the purpose of estimating the burden of residual effects, only those currently using a sleep medication $(n=1,605)$ were included. Those reporting residual sleep medication effects were compared to those reporting no residual effects, and the relationship between severity of residual effects and outcomes was modeled using regression. Subgroup analyses were conducted with patients aged 65 years or older due to reported vulnerability to adverse sleep medication effects [13].

Respondents were recruited primarily from previous respondents to the U.S. National Health and Wellness Survey (NHWS). The NHWS is a cross-sectional survey administered via the Internet to a sample of adults (18 years and older) who were identified through a web-based consumer survey panel. Members of the panel are recruited through opt-in emails, coregistration with other panels, e-newsletter campaigns, and online banner placements. All panelists explicitly agreed to become panel members, registered through unique email addresses, and completed in-depth demographic registration profiles. Invitations to participate in the NHWS were sent using a random stratified sampling framework to ensure the final sample of NHWS participants is representative of the adult population in the U.S. according to the Current Population Survey (CPS) of the U.S. Census (United States Bureau of the Census, 2012) in terms of age, gender, and white/non-white racial identification. Because of the size of the target sample and inclusion criteria of this study, additional respondents were also identified through the Light Speed Research Ailment Panel, which is composed of general panel members who have self-identified as having certain medical conditions.

Only those who were aged 18 years or older, self-reported a diagnosis of insomnia, and did not self-report diagnosis for sleep-disordered breathing, narcolepsy, shift work disorder, parasomnia, or other sleep condition were included in the survey. For inclusion in the current analysis, respondents also had to report current use of a prescription medication for sleep.

2.1. Procedure. Respondents were interviewed through a selfadministered, Internet-based questionnaire between December 5th and 18th, 2012. The protocol and questionnaire 
were reviewed and approved by Essex Institutional Review Board (Lebanon, New Jersey, USA) prior to recruitment of participants.

The presence and severity of residual effects were assessed through a series of items assessing to what extent the respondent experienced each of the nine side effects when they take their insomnia medication. These were feelings of drowsiness, difficulty concentrating, difficulty remembering, headaches, nausea, dry mouth, oversleeping, amnesia, and nightmares, using a Likert scale from 1 (None) to 5 ("Very Severe"). Drowsiness, difficulty concentrating, difficulty remembering, headaches, nausea, dry mouth, and oversleeping were also combined by summing the ratings of severity to provide an overall index of residual symptoms.

Respondents who indicated at least one residual symptom completed the Work Productivity and Activity Impairment, Specific Health Problem (WPAI-SHP) questionnaire [24]. The specific health problem was residual symptoms, and the term used in the instrument for residual symptoms was "next-day effects." Respondents were informed that this term was meant to indicate any side effects they feel the day after taking the medication. Four subscales (absenteeism, presenteeism, overall work impairment, and activity impairment) were generated in the form of percentages, with higher values indicating greater impairment. Absenteeism represents the percentage of work time missed due to next-day effects of sleep medication in the past seven days, and presenteeism represents the percentage of impairment in the past seven days while at work. Overall work impairment represents the overall amount of impact to work productivity due to either absenteeism or presenteeism (since they are mutually exclusive) in the past seven days. Activity impairment represents the percentage of impairment experienced during daily activities in the past seven days. Only employed respondents provided data on absenteeism, presenteeism, and overall work impairment but all respondents who reported at least one residual symptom rated their activity impairment.

Information on sleep difficulties was assessed using the Brief Insomnia Questionnaire (BIQ [25]). Information collected in the BIQ included in this analysis was the number of nights out of the past 7 with sleep problems due to trouble falling asleep, trouble staying asleep, trouble waking too early, waking feeling tired or unrested, and nights with at least one of these problems. The degree to which the individual's sleep problems interfered with home management, ability to work, social relationships, and close personal relationships was also assessed in the BIQ using a modified version of the Sheehan Disability Scales. These are scored from 0 to 10, with higher numbers indicating greater interference. A “don't know" response is also provided, and those who selected this option were excluded from analysis of the relevant item.

Insomnia Treatment History. A variety of items were used to characterize the respondents' treatment history for insomnia. These include the year diagnosed with insomnia, type of diagnosing doctor, type of prescribing doctor, type of doctor currently managing insomnia, whether the respondent is currently seeing a healthcare provider for insomnia, and previous prescription medications taken for insomnia.

Satisfaction with current medication was measured by the SATMED-Q [26]. This scale includes a total of 17 items that measure treatment satisfaction across multiple domains, including the presence and interference caused by side effects, the efficacy of the medication, convenience and ease of use, impact of medicine on everyday life, the follow-up from the doctor, and the patient's overall opinion of the medicine.

Health characteristics and risk behaviors incorporated in the analysis included body mass index (BMI; underweight, normal weight, overweight, and obese), alcohol consumption (consume alcohol versus abstain from alcohol), cigarette smoking (current smoker versus nonsmoker), and whether the person reports exercising vigorously in the past 30 days. The severity of respondents' comorbid medication conditions was assessed using the Charlson comorbidity index (CCI [27]). The CCI is calculated by weighting the presence of the following conditions and summing the result: HIV/AIDS, metastatic tumor, lymphoma, leukemia, any tumor, moderate/severe renal disease, hemiplegia, diabetes, mild liver disease, ulcer disease, connective tissue disease, chronic pulmonary disease, dementia, cerebrovascular disease, peripheral vascular disease, myocardial infarction, and congestive heart failure. The greater the total index score, the greater the comorbidity burden on the patient. Because insomnia commonly presents with psychiatric disorders, self-reported psychiatric diagnoses were also included in the questionnaire, including alcoholism, anxiety, bipolar disorder, depression, fibromyalgia, and schizophrenia.

2.2. Analysis. Analyses were conducted both on the full sample (aged 18 and older) and among the portion of the sample aged 65 years and older. Initial analyses compared those experiencing no residual symptoms to those who experienced at least one residual symptom using chi-square test for categorical variables and independent-samples $t$-tests for continuous variables. The relationship between the severity of residual effects and outcomes was also analyzed using multivariable regression. The multivariable models adjusted for covariates to reduce the likelihood that observed effects of residual symptoms were due to confounding factors. Covariates included gender (male versus female), race/ethnicity, age (continuous), BMI (overweight, obese, and missing versus normal/underweight), household income, comorbidity burden according to CCI, and a variety of psychiatric illnesses, which were found to be associated with residual symptoms during review of bivariate analyses. Models of treatment satisfaction were conducted using the total score from the SATMED-Q and were conducted using maximum likelihood linear regression. Likewise, ratings of disability were approximately normally distributed and also analyzed using linear models. Models of impairments measured by the WPAI were conducted using generalized linear models (GLMs) specifying a negative binomial distribution and a loglink function. All analyses were conducted first in the full sample and repeated in the subsample aged 65 and older. 


\section{Results}

Sample characteristics are presented in Table 1. Respondents were 52 years old on average, $78 \%$ were female and $87 \%$ were white. Most had their insomnia diagnosed and managed by a general practitioner. Psychiatric comorbidities were common among the sample, with approximately $50 \%$ reporting depression and approximately one-third of the sample reporting an anxiety disorder.

Approximately $80 \%$ of current users $(1,274 / 1,605)$ indicated some level of residual symptoms. Those reporting residual symptoms were slightly younger on average, but otherwise there were few demographic characteristics that differed across the presence of residual symptoms (Table 2). However, health characteristics differed according to presence of residual symptoms, with anxiety, depression, schizophrenia, and fibromyalgia all more likely among those with residual symptoms relative to those without residual symptoms, while alcoholism and bipolar disorder were marginally more likely. Psychiatrists were more often the diagnosing and prescribing doctor for those with residual symptoms than those without. The burden of comorbid conditions as represented by the CCI did not differ according to residual symptoms.

Problems with sleep in the prior 7 nights were common in current users of sleep medications. The presence of residual symptoms was associated with one additional day waking up tired/unrested, but not with the number of nights out of the past 7 with trouble falling asleep, staying asleep, or waking before the alarm (Table 3 ). In contrast, the impact of poor sleep on functioning was greater among those with residual effects, however, as ratings of interference in home management, ability to work, social relationships, and close relationships were all significantly higher among those reporting residual effects. Likewise, those who experienced residual symptoms were less satisfied with their current sleep medication than those who did not experience any residual symptoms (69.2 versus 76.0, $p<0.001$ ).

Correlational analyses confirmed that when present, severity of residual symptoms was associated with worse outcomes and lower satisfaction. All residual symptoms were significantly associated with greater ratings of work and activity impairment in bivariate correlations. Difficulty concentrating and drowsiness were particularly burdensome, which were correlated with work and activity impairment $r_{s}=.46-.49$. Likewise, the severity of difficulty concentrating $\left(r_{s}=-.347, p<0.001\right)$ and grogginess $\left(r_{s}=-.366, p<\right.$ 0.001 ) was most associated with (reduced) satisfaction with sleep medication (data not presented).

Regression analyses confirmed the association between residual symptoms and outcomes. The severity of residual symptoms was associated with lower satisfaction as measured by the SATMED-Q, (Beta $=-.37, p<0.001)$. The severity of residual symptoms was also associated with increased residual symptom-related work impairment, including absenteeism $(\mathrm{RR}=1.46,95 \% \mathrm{CI}: 1.34-1.60, p<0.001)$, presenteeism $(\mathrm{RR}=1.12$, 95\% CI: 1.09-1.14, $p<0.001)$, overall work impairment ( $\mathrm{RR}=1.13$, 95\% CI: $1.10-1.15, p<0.001)$, and impairment in nonwork activities ( $\mathrm{RR}=1.11$, 95\% CI: $1.10-$ $1.13, p<0.001)$. The severity of residual symptoms was also
TABLE 1: Respondent characteristics.

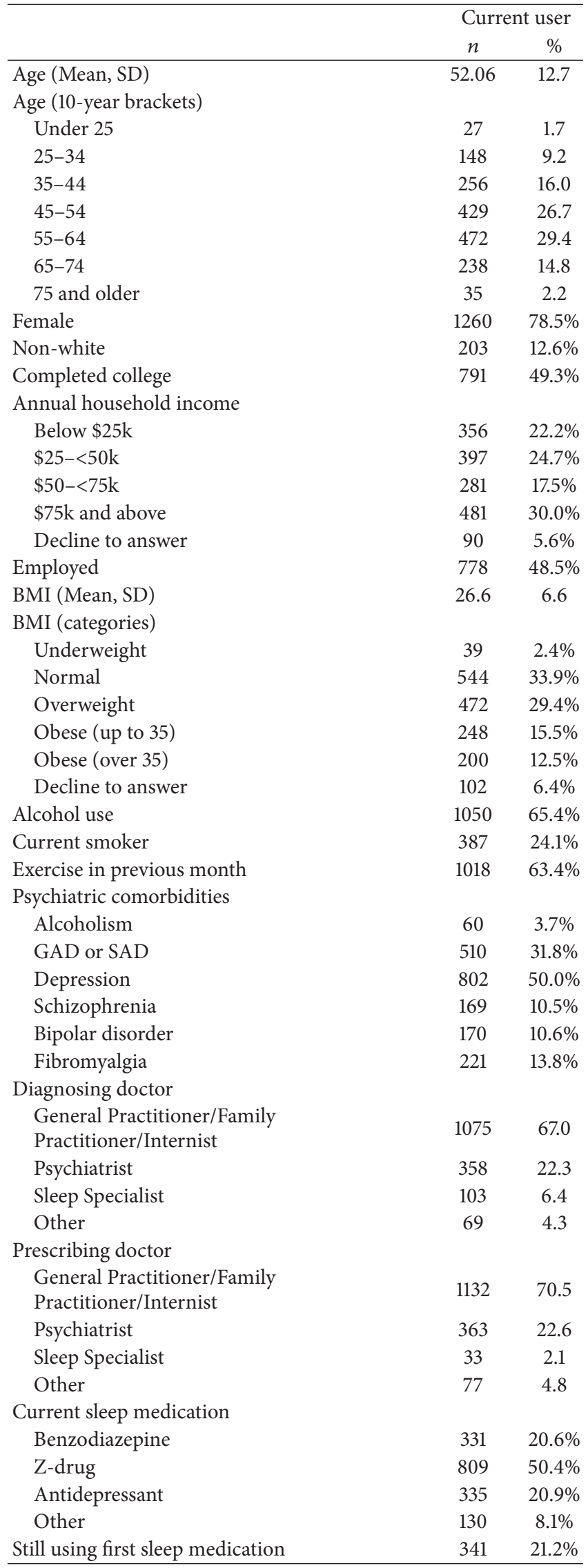


TABLE 2: Respondent characteristics by presence of residual symptoms.

\begin{tabular}{|c|c|c|c|c|c|}
\hline & \multicolumn{4}{|c|}{ Residual symptoms } & \multirow{3}{*}{$p$ value } \\
\hline & \multicolumn{2}{|c|}{ None $(N=331)$} & \multicolumn{2}{|c|}{ One or more $(N=1,274)$} & \\
\hline & $n$ & $\%$ & $n$ & $\%$ & \\
\hline Age (Mean, SD) & 54.1 & 12.7 & 51.5 & 12.6 & 0.001 \\
\hline Female & 259 & $78.2 \%$ & 1001 & $78.6 \%$ & 0.898 \\
\hline Non-white & 40 & $12.1 \%$ & 163 & $12.8 \%$ & 0.729 \\
\hline College degree & 227 & $68.6 \%$ & 862 & $67.7 \%$ & 0.750 \\
\hline Annual household income & & & & & 0.032 \\
\hline Below $\$ 25 \mathrm{k}$ & 73 & $22.1 \%$ & 283 & $22.2 \%$ & \\
\hline$\$ 25-<50 \mathrm{k}$ & 71 & $21.5 \%$ & 326 & $25.6 \%$ & \\
\hline$\$ 50-<75 k$ & 52 & $15.7 \%$ & 229 & $18.0 \%$ & \\
\hline$\$ 75 \mathrm{k}$ and above & 106 & $32.0 \%$ & 375 & $29.4 \%$ & \\
\hline Decline to answer & 29 & $8.8 \%$ & 61 & $4.8 \%$ & \\
\hline Employed & 153 & $46.2 \%$ & 625 & $49.1 \%$ & 0.358 \\
\hline BMI (Mean, SD) & 27.1 & 6.8 & 28.0 & 6.6 & 0.048 \\
\hline CCI (Mean, SD) & 0.60 & 1.11 & 0.77 & 1.36 & $0.032^{*}$ \\
\hline Alcohol use & 224 & $67.7 \%$ & 826 & $64.8 \%$ & 0.333 \\
\hline Current smoker & 75 & $22.7 \%$ & 312 & $24.5 \%$ & 0.488 \\
\hline Exercise in previous month & 207 & $62.5 \%$ & 811 & $63.7 \%$ & 0.706 \\
\hline \multicolumn{6}{|l|}{ Self-report psychiatric diagnoses } \\
\hline Alcoholism & 7 & $2.1 \%$ & 53 & $4.2 \%$ & 0.081 \\
\hline GAD or SAD & 72 & $21.8 \%$ & 438 & $34.4 \%$ & $<0.001$ \\
\hline Depression & 128 & $38.7 \%$ & 674 & $52.9 \%$ & $<0.001$ \\
\hline Schizophrenia & 23 & $6.9 \%$ & 146 & $11.5 \%$ & 0.017 \\
\hline Bipolar disorder & 26 & $7.9 \%$ & 144 & $11.3 \%$ & 0.069 \\
\hline Fibromyalgia & 31 & $9.4 \%$ & 190 & $14.9 \%$ & 0.009 \\
\hline Diagnosing doctor for insomnia & & & & & 0.002 \\
\hline General Practitioner/Family Practitioner/Internist & 240 & $72.5 \%$ & 835 & $65.5 \%$ & \\
\hline Psychiatrist & 50 & $15.1 \%$ & 308 & $24.2 \%$ & \\
\hline Sleep Specialist & 21 & $6.3 \%$ & 82 & $6.4 \%$ & \\
\hline Other & 20 & $6.0 \%$ & 49 & $3.8 \%$ & \\
\hline Prescribing doctor & & & & & 0.005 \\
\hline General Practitioner/Family Practitioner/Internist & 246 & $74.3 \%$ & 886 & $69.5 \%$ & \\
\hline Psychiatrist & 54 & $16.3 \%$ & 309 & $24.3 \%$ & \\
\hline Sleep Specialist & 11 & $3.3 \%$ & 22 & $1.7 \%$ & \\
\hline Other & 20 & $6.0 \%$ & 57 & $4.5 \%$ & \\
\hline
\end{tabular}

Note: $*$ indicates Welch's test was used in lieu of parametric $t$-test due to nonhomogeneity of variance.

TABLE 3: Sleep-related trouble according to the presence of residual symptoms.

\begin{tabular}{|c|c|c|c|c|c|}
\hline & \multicolumn{4}{|c|}{ Residual symptoms } & \multirow{3}{*}{$p$ value } \\
\hline & \multicolumn{2}{|c|}{ None $(N=331)$} & \multicolumn{2}{|c|}{ One or more $(N=1,274)$} & \\
\hline & Mean & SD & Mean & SD & \\
\hline Nights out of 7 with trouble falling asleep & 5.1 & 2.3 & 5.3 & 2.0 & $0.297^{*}$ \\
\hline Nights out of 7 with trouble staying asleep & 5.4 & 2.2 & 5.4 & 2.2 & 0.856 \\
\hline Number of days out of 7 waking before alarm & 4.6 & 2.6 & 4.5 & 2.5 & 0.253 \\
\hline Number of days out of 7 waking tired/unrested & 4.6 & 2.4 & 5.6 & 1.9 & $<0.001^{*}$ \\
\hline Nights out of 7 with any problem above & 6.0 & 1.5 & 6.1 & 1.5 & 0.891 \\
\hline Sleep problems interfere with home management & 3.9 & 3.0 & 5.6 & 2.8 & $<0.0001$ \\
\hline Sleep problems interfere with ability to work & 3.1 & 3.1 & 4.6 & 3.2 & $<0.0001$ \\
\hline Sleep problems interfere with social relationships & 3.6 & 3.2 & 5.3 & 3.0 & $<0.0001$ \\
\hline Sleep problems interfere with close relationships & 3.3 & 3.2 & 5.2 & 3.1 & $<0.0001$ \\
\hline
\end{tabular}

Note: $*$ indicates Welch's test was used in lieu of parametric $t$-test due to nonhomogeneity of variance. 
TABLE 4: Respondent characteristics by experience of residual symptoms in respondents aged 65 and older.

\begin{tabular}{|c|c|c|c|c|c|}
\hline & \multicolumn{4}{|c|}{ Residual symptoms } & \multirow{3}{*}{$p$ value } \\
\hline & \multicolumn{2}{|c|}{ None $(N=78)$} & \multicolumn{2}{|c|}{ One or more $(N=195)$} & \\
\hline & $n$ & $\%$ & $n$ & $\%$ & \\
\hline Age (Mean, SD) & 69.37 & 4.07 & 69.72 & 4.53 & 0.558 \\
\hline Female & 63 & $80.8 \%$ & 143 & $73.3 \%$ & 0.197 \\
\hline Non-white & 4 & $5.1 \%$ & 8 & $4.1 \%$ & 0.709 \\
\hline Completed college & 59 & $75.6 \%$ & 145 & $71.4 \%$ & 0.466 \\
\hline Annual household income & & & & & 0.076 \\
\hline Below $\$ 25 \mathrm{k}$ & 13 & $16.7 \%$ & 32 & $16.4 \%$ & \\
\hline$\$ 25-<50 \mathrm{k}$ & 13 & $16.7 \%$ & 61 & $31.3 \%$ & \\
\hline$\$ 50-<75 k$ & 14 & $17.9 \%$ & 34 & $17.4 \%$ & \\
\hline$\$ 75 \mathrm{k}$ and above & 26 & $33.3 \%$ & 53 & $27.2 \%$ & \\
\hline Decline to answer & 12 & $15.4 \%$ & 15 & $7.7 \%$ & \\
\hline Employed & 15 & $19.2 \%$ & 33 & $16.9 \%$ & 0.651 \\
\hline BMI (categories) & & & & & 0.968 \\
\hline Underweight & 3 & $3.8 \%$ & 6 & $3.1 \%$ & \\
\hline Normal & 26 & $33.3 \%$ & 66 & $33.8 \%$ & \\
\hline Overweight & 28 & $35.9 \%$ & 65 & $33.3 \%$ & \\
\hline Obese (up to 35 ) & 11 & $14.1 \%$ & 34 & $17.4 \%$ & \\
\hline Obese (over 35) & 7 & $9.0 \%$ & 19 & $9.7 \%$ & \\
\hline Refused & 3 & $3.8 \%$ & 5 & $2.6 \%$ & \\
\hline Alcohol use & 51 & $65.4 \%$ & 133 & $68.2 \%$ & 0.653 \\
\hline Smokes & 13 & $16.7 \%$ & 23 & $11.8 \%$ & 0.282 \\
\hline Exercise in previous month & 43 & $55.1 \%$ & 113 & $57.9 \%$ & 0.671 \\
\hline \multicolumn{6}{|l|}{ Psychiatric comorbidities } \\
\hline Alcoholic & 1 & $1.3 \%$ & 6 & $3.1 \%$ & 0.397 \\
\hline Anxiety & 8 & $10.3 \%$ & 45 & $23.1 \%$ & 0.016 \\
\hline Depression & 18 & $23.1 \%$ & 76 & $39.0 \%$ & 0.013 \\
\hline Schizophrenia & 1 & $1.3 \%$ & 33 & $16.9 \%$ & 0.000 \\
\hline Bipolar disorder & 3 & $3.8 \%$ & 5 & $2.6 \%$ & 0.570 \\
\hline Fibromyalgia & 4 & $5.1 \%$ & 31 & $15.9 \%$ & 0.016 \\
\hline Diagnosing doctor & & & & & 0.074 \\
\hline General Practitioner/Family Practitioner/Internist & 66 & $84.6 \%$ & 142 & $72.8 \%$ & \\
\hline Psychiatrist & 5 & $6.4 \%$ & 29 & $14.9 \%$ & \\
\hline Sleep Specialist & 2 & $2.6 \%$ & 15 & $7.7 \%$ & \\
\hline Other & 5 & $6.4 \%$ & 9 & $4.6 \%$ & \\
\hline Prescribing doctor & & & & & 0.264 \\
\hline General Practitioner/Family Practitioner/Internist & $82.1 \%$ & 162 & $83.1 \%$ & $82.1 \%$ & \\
\hline Psychiatrist & $7.7 \%$ & 24 & $12.3 \%$ & $7.7 \%$ & \\
\hline Sleep Specialist & $3.8 \%$ & 3 & $1.5 \%$ & $3.8 \%$ & \\
\hline Other & $6.4 \%$ & 6 & $3.1 \%$ & $6.4 \%$ & \\
\hline
\end{tabular}

Note: $*$ indicates Welch's test was used in lieu of parametric $t$-test due to nonhomogeneity of variance.

associated with increases in sleep-related interference on the four domains measured in the BIQ in the regression analyses; home management $($ Beta $=.31, p<0.001)$, ability to work $($ Beta $=.31, p<0.001)$, social relationships, $($ Beta $=.32$, $p<0.001$ ), and close personal relationships (Beta $=.30$, $p<0.001)$ were all similarly affected.

Analysis of those aged 65 and older also revealed a high proportion (71\%; 195 of 273) of current users reporting at least one residual symptom. As in the full sample, the rates of anxiety, depression, schizophrenia, and fibromyalgia were higher among those with residual symptoms (Table 4). Unlike the full sample, patients aged 65 or older with residual symptoms had higher CCI scores relative to those without residual symptoms.

Results of comparisons of sleep outcomes also mirrored those of the full sample (Table 5). The number of nights with different types of sleep problems were comparable across those with and without residual symptoms except for 
TABLE 5: Sleep-related trouble according to the presence of residual symptoms in respondents 65 years and older.

\begin{tabular}{|c|c|c|c|c|c|}
\hline & & Resid & oms & & \\
\hline & Non & & One o & 195) & $p$ value \\
\hline & Mean & SD & Mean & SD & \\
\hline Nights out of 7 with trouble falling asleep & 4.6 & 2.5 & 5.2 & 2.1 & $0.059^{*}$ \\
\hline Nights out of 7 with trouble staying asleep & 5.4 & 2.2 & 5.4 & 2.2 & 0.822 \\
\hline Number of days out of 7 wake up before alarm & 4.6 & 2.7 & 4.3 & 2.7 & 0.461 \\
\hline Number of days out of 7 wake up tired/unrested & 3.7 & 2.7 & 4.9 & 2.4 & $0.001^{*}$ \\
\hline Nights out of 7 with problem & 5.9 & 1.6 & 5.8 & 1.8 & 0.651 \\
\hline Sleep problems interfere with home management & 2.7 & 2.8 & 4.6 & 2.8 & $<0.001$ \\
\hline Sleep problems interfere with ability to work & 1.7 & 2.5 & 3.0 & 3.0 & $0.001^{*}$ \\
\hline Sleep problems interfere with social relationships & 2.6 & 3.0 & 3.9 & 2.8 & 0.001 \\
\hline Sleep problems interfere with close relationships & 2.5 & 3.1 & 3.5 & 2.9 & 0.010 \\
\hline
\end{tabular}

Note: $*$ indicates Welch's test was used in lieu of parametric $t$-test due to nonhomogeneity of variance.

days waking up tired or unrested. Also consistent with the full sample, levels of disability due to sleep problems were elevated in those with residual symptoms relative to those without for all four domains measured. Those who experienced at least one residual symptom also had marginally lower satisfaction than those without any residual symptoms (74.7 versus $78.5, p=0.057$ ).

As in the full sample, the expected relationship between residual symptoms and satisfaction with sleep medication was seen in the correlations between satisfaction and ratings of individual residual symptoms (data not presented). Difficulty concentrating was most closely related to satisfaction $\left(r_{s}=-.34, p<0.001\right)$. Total residual symptoms and difficulty concentrating were most closely related to sleep medicationrelated impairment to nonwork activities (both $r_{s}=.46$, $p<0.001$ ).

Regression results demonstrated that total residual symptoms were associated with lower satisfaction with current medication among those aged 65 years and older (Beta $=$ $-.37, p<0.001)$. The severity of residual symptoms was also associated with increases in sleep-related interference on home management (Beta $=.30, p<0.001)$, social relationships (Beta $=.26, p<0.001$ ), and close personal relationships (Beta $=.27, p<0.001$ ). Total residual symptoms were also associated with impairment to nonwork activities on the WPAI ( $\mathrm{RR}=1.18,95 \% \mathrm{CI}: 1.11-1.25, p<0.001$ ).

\section{Discussion}

This study described the relationship between perceived residual sleep medication effects and a wide range of important outcomes for insomnia patients. This was the first study, to our knowledge, to describe the magnitude of the relationship between residual sleep medication effects and this large array of patient-reported outcomes, particularly in a single, large sample. Findings are particularly novel for the older patients, as the literature focuses primarily on what the residual effects are, rather than their correlates, for this demographic group.
Residual medication effects-such as feelings of being drowsy, groggy, or sluggish the next day, difficulty concentrating/remembering, or sleeping too much-were reported by approximately four out of every five individuals currently using prescription sleep medication. Overall, findings showed significant burden experienced by patients reporting residual sleep medication effects relative to those not reporting such effects.

Though patients with and without perceived residual effects suffered a similar number of nights with sleep problems (falling asleep, staying asleep, waking before the alarm, or any problem), the experience of residual effects was associated with an average of one more day per week of "unrestful sleep." One potential explanation is that the residual effects of the sleep medication itself are responsible for the difference, though this is only speculation; the present analysis was not designed to identify the cause. Patients reporting residual effects were also less satisfied with their medications. Moreover, there were clear relationships between increasingly severe residual symptoms and decreased satisfaction, as well as increasingly severe residual symptoms and greater work and activity impairment, and greater sleep-related interference in home management, ability to work, and social relationships. Though respondents reporting residual effects indicated they experienced more psychiatric symptomatology and other comorbidities than those not experiencing such effects, the relationships between functioning and residual symptom severity remained significant after these and other relevant covariates were accounted for.

Analysis of older patients showed a similar pattern of relationships. Differences between those with and without residual symptoms were only marginal, but the correlation between increasingly severe residual symptoms and decreased satisfaction was of considerable magnitude. Increasing symptom severity corresponded with greater impairment across residual symptom-related nonwork activities, home management, ability to work, and social relationships. These relationships held when relevant covariates were included as well. 
In support of prior research, insomnia patients experiencing residual symptoms comprise a group who are under particular strain, even relative to other already-burdened insomnia patients. This study uniquely describes the depth of this strain, which appears to occur across a wide range of domains and is likely affecting patients' global functioning and quality of life. Increasing residual symptom severity appears to affect level of impairment. Regarding financial burden, the strain could be indirectly affecting the work force and healthcare system. As hypothesized, older patients experiencing residual sleep medication effects showed the additional burden of more medical comorbidities. The comorbid conditions could potentially be aggravated or exacerbated by sleep medication side effects.

There are a number of limitations of the current study that should be considered alongside the results. Most importantly, this was an observational study, and the correlational nature of the data collection precludes any causal attribution. Likewise, the cross-sectional design does not allow us to ascertain whether the residual symptoms precede difficulties in home management, ability to work, and so forth, or whether residual symptoms occur in response to a worsening of such problems. Indeed, some residual symptoms, such as grogginess and difficulty concentrating, are also symptoms of insomnia, so some of the residual symptoms reported here may instead be symptoms of inadequately treated insomnia rather than next-day effects of sleep medication or a combination of both inadequate efficacy and medication side effects. Residual effects were self-reported rather than using objective measures of attention, memory, or reaction time. Another study limitation includes the margin of error inherent in any study using self-report measures, though insomnia itself can only be diagnosed via self-report, making self-report vital to this study [11]. Finally, the residual sleep medication effects we reported likely relate to other medical, psychosocial, quality of life, and economic outcomes that we did not measure. We may thus be underestimating the true extent of humanistic and economic burden.

\section{Conclusions}

Ultimately, patients who experience residual sleep medication effects represent a group with significant impairment of workplace, home, and social life activities; as the perceived severity of the residual symptoms increases, so does the burden. Thus, thorough medical and psychosocial/behavioral assessment of individuals experiencing residual effects is recommended (especially for the elderly). Also, improved management of insomnia would be beneficial. Behavioral and cognitive interventions have essentially no side effects and have been shown to lead to long-lasting, sustained improvements in sleep symptoms and parameters over 6 months to 24 months [28]. However, the degree of sleep medication use in this sample demonstrates that many may prefer, or need, pharmacotherapy for insomnia, highlighting a need for medications with fewer residual symptoms. The development of sleep medications with reduced residual effect profiles will be important for treatment of this patient population.

\section{Conflict of Interests}

This study and the preparation of the paper were conducted by Kantar Health with funds from Merck \& Co., Inc. Timothy Fitzgerald is an employee of Merck \& Co., Inc., and may own Merck \& Co., Inc., stock. Jeffrey Vietri is an employee of Kantar Health.

\section{Acknowledgments}

This study was conducted by Kantar Health with funding from Merck \& Co., Inc. Timothy Fitzgerald is an employee of Merck \& Co., and Jeffrey Vietri is an employee of Kantar Health. The authors would like to acknowledge Lewis Kopenhafer for his role in the construction of the questionnaire and Tammy Schuler, Ph.D., for her editorial assistance in the preparation of the paper.

\section{References}

[1] C. M. Morin, M. LeBlanc, M. Daley, J. P. Gregoire, and C. Mérette, "Epidemiology of insomnia: prevalence, self-help treatments, consultations, and determinants of help-seeking behaviors," Sleep Medicine, vol. 7, no. 2, pp. 123-130, 2006.

[2] M. M. Ohayon and C. F. Reynolds III, "Epidemiological and clinical relevance of insomnia diagnosis algorithms according to the DSM-IV and the International Classification of Sleep Disorders (ICSD)," Sleep Medicine, vol. 10, no. 9, pp. 952-960, 2009.

[3] C. M. Morin, L. Bélanger, M. LeBlanc et al., "The natural history of insomnia a population-based 3-year longitudinal study," Archives of Internal Medicine, vol. 169, no. 5, pp. 447-453, 2009.

[4] H. Morphy, K. M. Dunn, M. Lewis, H. F. Boardman, and P. R. Croft, "Epidemiology of insomnia: a longitudinal study in a UK population," Sleep, vol. 30, no. 3, pp. 274-280, 2007.

[5] J. E. Aikens and M. E. Rouse, "Help-seeking for insomnia among adult patients in primary care," Journal of the American Board of Family Practice, vol. 18, no. 4, pp. 257-261, 2005.

[6] N. J. Pearson, L. L. Johnson, and R. L. Nahin, "Insomnia, trouble sleeping, and complementary and alternative medicine: analysis of the 2002 national health interview survey data," Archives of Internal Medicine, vol. 166, no. 16, pp. 1775-1782, 2006.

[7] T. Roth, S. Jaeger, R. Jin, A. Kalsekar, P. E. Stang, and R. C. Kessler, "Sleep problems, comorbid mental disorders, and role functioning in the national comorbidity survey replication," Biological Psychiatry, vol. 60, no. 12, pp. 1364-1371, 2006.

[8] M. T. Smith and J. A. Haythornthwaite, "How do sleep disturbance and chronic pain inter-relate? Insights from the longitudinal and cognitive-behavioral clinical trials literature," Sleep Medicine Reviews, vol. 8, no. 2, pp. 119-132, 2004.

[9] M. Suka, K. Yoshida, and H. Sugimori, "Persistent insomnia is a predictor of hypertension in Japanese male workers," Journal of Occupational Health, vol. 45, no. 6, pp. 344-350, 2003.

[10] A. N. Vgontzas, D. Liao, S. Pejovic et al., "Insomnia with short sleep duration and mortality: the Penn State cohort," Sleep, vol. 33, no. 9, pp. 1159-1164, 2010.

[11] S. L. Schutte-Rodin, L. Broch, D. Buysee, C. Dorsey, and M. Sateia, "Clinical guideline for the evaluation and management of chronic insomnia in adults," Journal of Clinical Sleep Medicine, vol. 4, no. 5, pp. 487-504, 2008. 
[12] C. E. Reeder, M. Franklin, and T. J. Bramley, "Current landscape of insomnia in managed care," American Journal of Managed Care, vol. 13, supplement 5, pp. S112-S116, 2007.

[13] C. M. Morin and R. Benca, "Chronic insomnia," The Lancet, vol. 379, no. 9821, pp. 1129-1141, 2012.

[14] A. J. Roth, W. V. Mccall, and A. Liguori, "Cognitive, psychomotor and polysomnographic effects of trazodone in primary insomniacs," Journal of Sleep Research, vol. 20, no. 4, pp. 552558, 2011.

[15] J. S. Poceta, "Zolpidem ingestion, automatisms, and sleep driving: a clinical and legal case series," Journal of Clinical Sleep Medicine, vol. 7, no. 6, pp. 632-638, 2011.

[16] M.-J. Tsai, Y.-H. Tsai, and Y.-B. Huang, "Compulsive activity and anterograde amnesia after zolpidem use," Clinical Toxicology, vol. 45, no. 2, pp. 179-181, 2007.

[17] A. M. Holbrook, R. Crowther, A. Lotter, C. Cheng, and D. King, "Meta-analysis of benzodiazepine use in the treatment of insomnia," Canadian Medical Association Journal, vol. 162, no. 2, pp. 225-233, 2000.

[18] G. K. Zammit, B. Corser, K. Doghramji et al., "Sleep and residual sedation after administration of zaleplon, zolpidem, and placebo during experimental middle-of-the-night awakening," Journal of Clinical Sleep Medicine, vol. 2, no. 4, pp. 417-423, 2006.

[19] A. Y. Avidan, B. E. Fries, M. L. James, K. L. Szafara, G. T. Wright, and R. D. Chervin, "Insomnia and hypnotic use, recorded in the minimum data set, as predictors of falls and hip fractures in Michigan nursing homes," Journal of the American Geriatrics Society, vol. 53, no. 6, pp. 955-962, 2005.

[20] S. Wilson and S. Argyropoulos, "Antidepressants and sleep: a qualitative review of the literature," Drugs, vol. 65, no. 7, pp. 927947, 2005.

[21] J. C. Verster and T. Roth, "Gender differences in highway driving performance after administration of sleep medication: a review of the literature," Traffic Injury Prevention, vol. 13, no. 3, pp. 286292, 2012.

[22] J. Glass, K. L. Lanctôt, N. Herrmann, B. A. Sproule, and U. E. Busto, "Sedative hypnotics in older people with insomnia: metaanalysis of risks and benefits," The British Medical Journal, vol. 331, no. 7526, article 1169, 2005.

[23] S. D. Berry, Y. Lee, S. Cai, and D. D. Dore, "Nonbenzodiazepine sleep medication use and hip fractures in nursing home residents," JAMA Internal Medicine, vol. 173, no. 9, pp. 754-761, 2013.

[24] M. C. Reilly, A. S. Zbrozek, and E. M. Dukes, "The validity and reproducibility of a work productivity and activity impairment instrument," PharmacoEconomics, vol. 4, no. 5, pp. 353-365, 1993.

[25] R. C. Kessler, C. Coulouvrat, G. Hajak et al., "Reliability and validity of the brief insomnia questionnaire in the America Insomnia Survey," Sleep, vol. 33, no. 11, pp. 1539-1549, 2010.

[26] M. A. Ruiz, A. Pardo, J. Rejas, J. Soto, F. Villasante, and J. L. Aranguren, "Development and validation of the 'treatment satisfaction with medicines questionnaire' (SATMED-Q)," Value in Health, vol. 11, no. 5, pp. 913-926, 2008.

[27] M. E. Charlson, P. Pompei, K. L. Ales, and C. R. MacKenzie, "A new method of classifying prognostic comorbidity in longitudinal studies: development and validation," Journal of Chronic Diseases, vol. 40, no. 5, pp. 373-383, 1987.

[28] O. C. Ioachimescu and A. A. El-Solh, "Pharmacotherapy of insomnia," Expert Opinion on Pharmacotherapy, vol. 13, no. 9, pp. 1243-1260, 2012. 


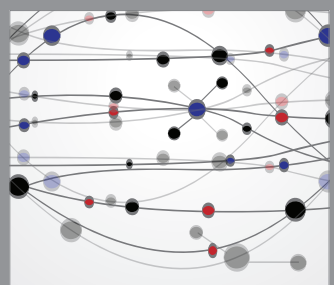

The Scientific World Journal
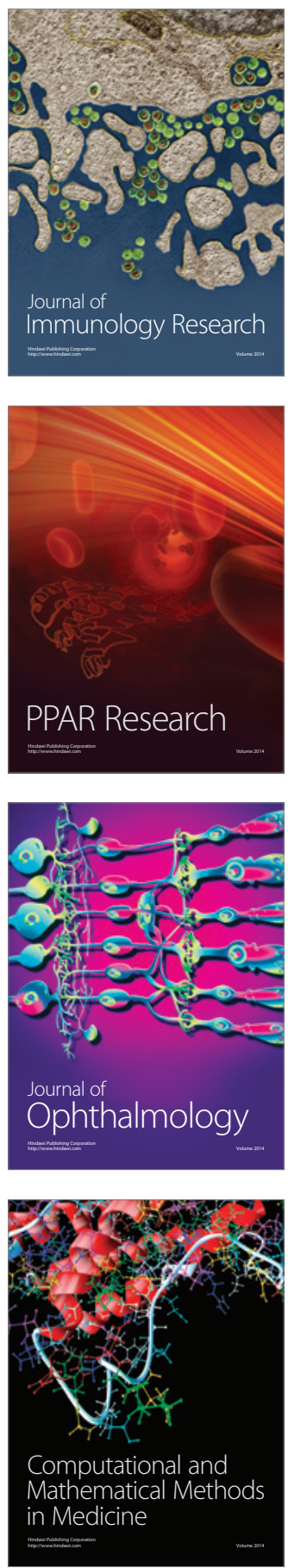

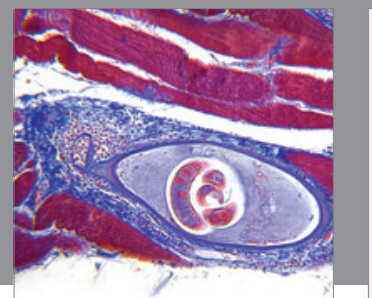

Gastroenterology

Research and Practice
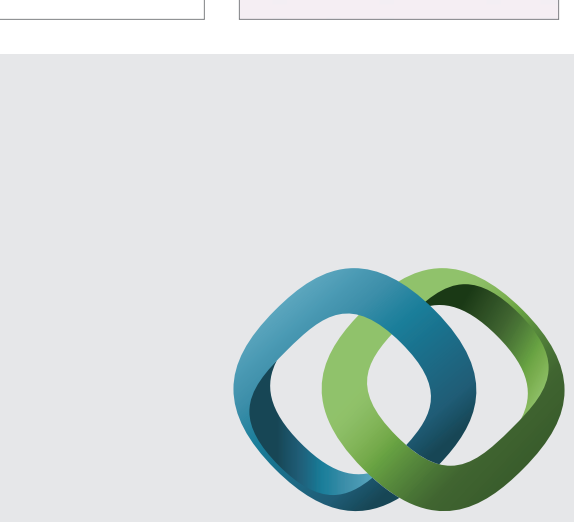

\section{Hindawi}

Submit your manuscripts at

http://www.hindawi.com
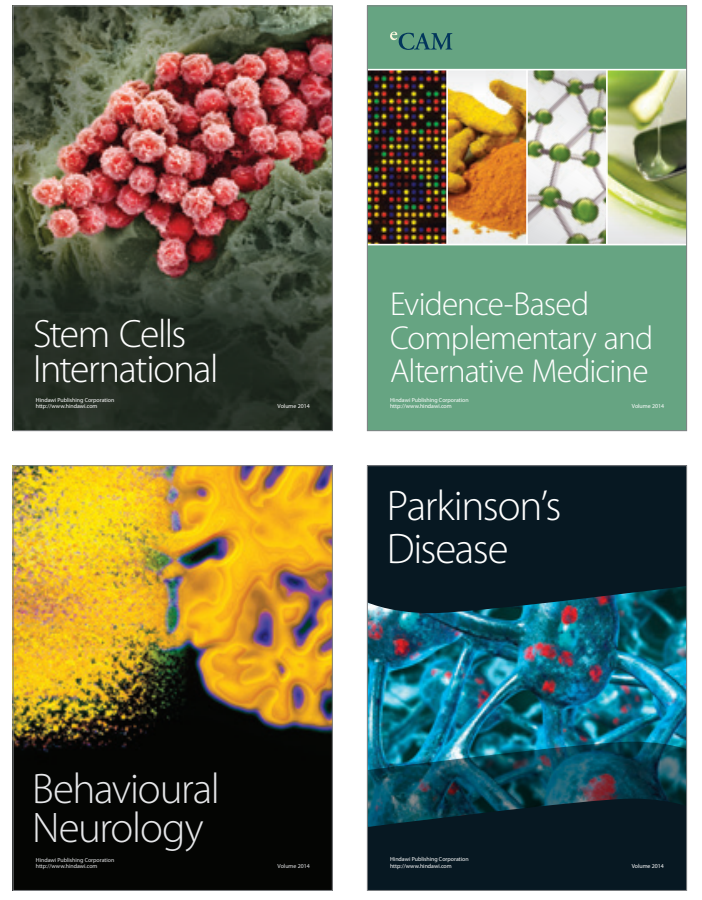
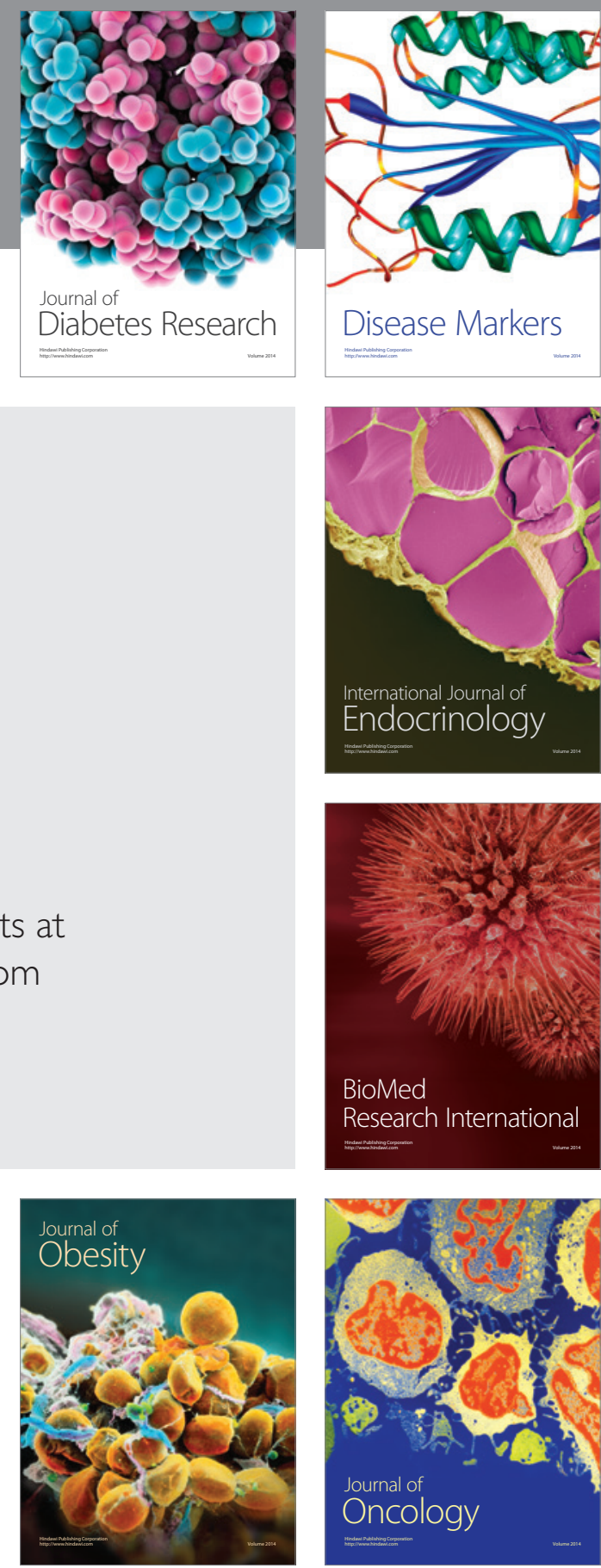

Disease Markers
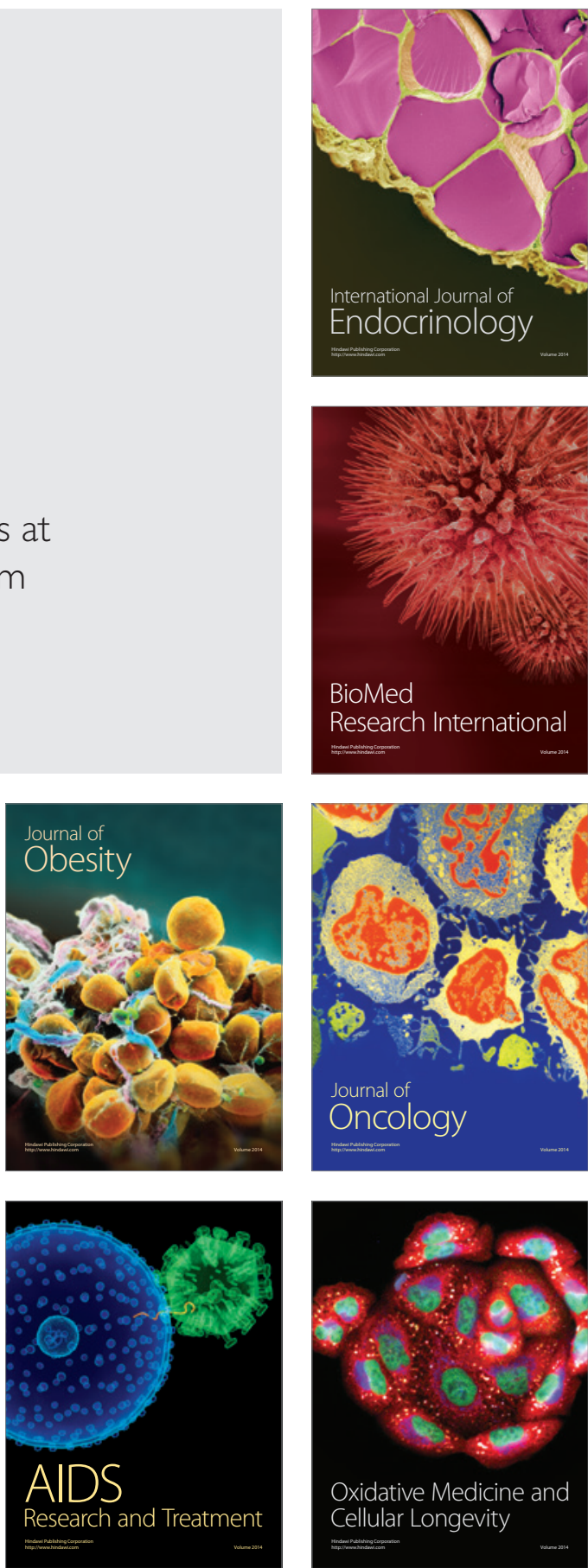